Methods During July-August, 2009, a questionnaire survey of stratified cluster sampling was conducted among 7421 permanent residents over 18 years old in 4 Beijing districts. The responders had an age range of $20-89$ years old.

Results (1) In 2009, the smoking rate of urban and rural Beijing residents was $24.98 \%$. The smoking rate of rural population was higher than that of urban counterpart (urban $15.41 \%$, urban-rural $21.20 \%$ \& rural $29.73 \%$ ). The smoking rate was higher in males than females $(57.18 \%$ vs $7.43 \%)$. (2) Overall speaking, the smoking rate was higher in those with a lower educational level than those with a higher educational level. The smoking rate tended to be different with the advancing ages in male and female populations. It increased with the advancing age in males and decreased with the advancing age in females. The magnitude of smoking rate had no obvious correlation with the undertaken occupations. (3) Analysis of anti-smoking urge: Disease prevention accounted for $52.65 \%$, morbidity $22.57 \%$, family objection $18.14 \%$, environmental limitation $5.95 \%$, promotion \& education exhibition $5.75 \%$ and physician advising $3.98 \%$. The major anti-smoking-related factors include cardiocerebrovascular sequel, diabetes, dyslipidemia and attending health workshops.

Conclusion As compared with the results of previous surveys, the smoking rate decreased slightly in the Beijing urban residents. The major aspect of smoking-quitting urge is disease prevention. And the anti-smoking education should be strenuously intensified.

\section{E0294 SURVEY OF EPIDEMIOLOGICAL CHARACTERISTICS OF MAJOR CHRONIC DISEASES IN URBAN AND RURAL BEIJING RESIDENTS OVER 18 YEARS OLD IN 2009}

doi:10.1136/hrt.2010.208967.294

${ }^{1}$ RongJing Ding, ${ }^{2}$ Jinming Yu, ${ }^{2}$ Lijun Zhang, ${ }^{1}$ Dayi Hu. ${ }^{1}$ Heart and Vascular Center, Beijing University People's Hospital, Beijing 100044, China; 'Epidemiology and Statistics Department, Epidemiology and Statistics Department, Fudan University, ShangHai, China

Objective To understand the epidemiological characteristics and control levels of major chronic diseases in Beijing municipality in 2009.

Methods During July-August 2009, a questionnaire survey of stratified cluster sampling was conducted among 7421 permanent residents over 18 years old in 4 Beijing districts. The surveying method included questionnaire, physical examination and laboratory testing.

Results The rates of morbidity, awareness, medication and control for hyperlipidaemia were $53.97 \%, 28.05 \%, 13.05 \%$ and $10.03 \%$ respectively. Those for hypertension were $52.33 \%, 64.29 \%, 57.53 \%$ and $19.9 \%$ respectively. For diabetes, $13.63 \%, 71.23 \%, 68.39 \%$ and $32.73 \%$. The morbidity rates of hypertension and diabetes increased with the advancing age, the morbidity of hyperlipidaemia decreased with the advancing age, and were all higher in the urban population than in the urban population. The rates of awareness, treatment and control for major chronic diseases were all higher in the urban population than the rural counterpart and were lower in the youth than the elders. The morbidity rates of lower extremity atherosclerosis, coronary heart disease and stroke were $11.0 \%, 6.0 \%$ and $4.4 \%$ respectively.

Conclusion As compared with the results of previous surveys, in both urban and rural areas, the morbidity rates of major chronic diseases increased markedly in permanent Beijing residents over 18 years old in 2009. The morbidity level of major chronic diseases remains higher in the urban population than in the rural counterpart. The youth has a markedly elevated morbidity rate of chronic diseases. The control of chronic diseases should be implemented in both urban and rural Beijing. And the focus is placed upon the young individuals.

\section{Q0295 TREND COMPARISON OF MAJOR CHRONIC DISEASES IN URBAN AND RURAL BEIJING AREAS DURING 2007-2009}

doi:10.1136/hrt.2010.208967.295

${ }^{1}$ Rong Jing Ding, ${ }^{2}$ Jinming Yu, ${ }^{2}$ Lijun Zhang, ${ }^{1}$ Dayi Hu. ${ }^{1}$ Heart and Vascular Center,

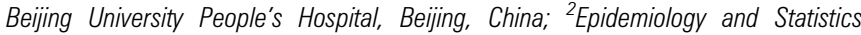
Department, Fudan University, ShangHai, China

Objective To evaluate and compare the trends of major chronic diseases in urban and rural Beijing areas during 2007-2009.

Methods During July-August in 2007 and 2009, a cross-sectional survey of stratified cluster sampling was conducted respectively among 10002 and 7421 permanent residents over 18 years old in 4 Beijing districts. The surveying method included questionnaire, physical examination and laboratory testing.

Results Within the 2-year period, the prevalence of hyperlipidaemia, hypertension and diabetes in urban and rural Beijing populations increased by $2.32 \%, 7.75 \%$ and $2.59 \%$ respectively. The awareness rate increased by $5.55 \%, 1.5 \%$ and $9.36 \%$ respectively. Except for a decline of $3.32 \%$ for hyperlipidaemia, the treatment rate increased by $2.77 \%$ and $8.61 \%$ respectively. The control rate had not a marked increase, but there was a declining trend of $1.22 \%,-4.54 \%$ and $0.67 \%$ respectively. Within the last 2 years, mean total cholesterol decreased by $0.302 \mathrm{mmol} / \mathrm{l}$, mean low-density lipoprotein and mean body mass index increased by $0.419 \mathrm{mmol} / 1$ and $0.455 \mathrm{~kg} / \mathrm{m}^{2}$ respectively. The increment was higher in females than males. The smoking rate declined by a percentage of 1.73 . The prevalence of lower extremity atherosclerosis increased from $4.65 \%$ to $11.0 \%$. With a fourfold increment in rural population, it was higher in females than males. The total prevalence of coronary heart disease and stroke didn't made any changes within 2 years. But there were difference and change patterns about the prevalence of $\mathrm{CHD}$ and stroke between urban and rural areas.

Conclusion There has been an upward trend in the prevalence of major chronic diseases in urban and rural Beijing residents over the last 2 years. With a low control rate, the urban population has a higher prevalence than the rural counterpart. Besides education promotion, the intervention efforts for chronic diseases in Beijing urban and rural areas should be strengthened.

\section{e0296 COMMUNITY-HOSPITAL CLASSIFICATION MANAGEMENT MODEL RESULTS}

doi:10.1136/hrt.2010.208967.296

${ }^{1}$ Xin Zhao, ${ }^{2}$ Ying-Jue Xu, ${ }^{1}$ Rui-Jie Li. ${ }^{1}$ Second Hospital of Chaoyang District, Beijing; ${ }^{2}$ North China Coal Medical College

Background Hypertension is a common disease that seriously endangers the health of populations, frequently-occurring disease, is one of stroke, coronary heart disease the major risk factors. At present, China in the prevention and treatment of hypertension, the "three low, three high, three errors", namely, (three high: prevalence rate, high morbidity and mortality is high; three low: low awareness rate, treatment rate is low, the control rate is low; three errors: do not want medication, do not tolerate no medication, no medication by your doctor) the situation has not significantly improved, the low rate of effective control of hypertension phenomenon is a widespread problem. At home and abroad a large number of studies have shown that people in the community to implement the classification of hypertension management is the crowd the key to prevention and treatment of cardiovascular and cerebrovascular diseases. Current hypertension management at different levels of remaining on the following questions, (1) Comprehensive chronic disease prevention and control capacity of the community vacancy. Community Health Workers of knowledge, lack of technical skills, 